\title{
Constraints on Photosynthesis on Earth and Earth-Like Planets
}

\author{
John A. Raven \\ Biological Sciences Institute, University of Dundee, U.K. \\ Ramon D. Wolstencroft \\ Royal Observatory, Edinburgh EH9 3HJ, U.K.
}

\begin{abstract}
We discuss how the spectral mismatch between solar radiation at the Earth's surface and the two absorption maxima (440 and $680 \mathrm{~nm}$ ) of the dominant chlorophyll pigments can be explained as an evolutionary accident of the marine habitat in which photosynthesis evolved on Earth. The geometric rigidity and great stability of chlorophyll make it a likely "universal pigment"; given this, together with the spectral absorption properties of water, the evolution of photosynthesis on Earth-like planets (ELPs) orbiting in the habitable zones of A, F and G stars should be relatively straightforward. However for ELPs orbiting cool K and M stars, the effects of variable UV emission from the ELP at earlier epochs and the high absorption of red light by water could lead to a "feast and famine" existence for photosynthetic organisms in the ELP ocean. Full exploitation of the cool star radiation when UV activity has declined may lead to an evolution of the photosynthetic machinery from the terrestrial two photon to a three photon system.
\end{abstract}

\section{Introduction}

Photosynthesis on Earth involves a relatively small range of chemical structures for the pigments that absorb light and perform photochemistry. These structures are cyclic or linear tetrapyrroles (also known as porphyrins) such as chlorophylls, bacteriochlorophylls and phycobilins, and the polyisoprene carotenoids. In intact cells the chlorophylls and bacteriochorophylls and the carotenoids have an absorption band peaking in the 420-550 nm range, and the chlorophylls and bacteriochorophylls have another absorption band peaking in the $600-1000 \mathrm{~nm}$ range. All of the photochemistry of (bacterio-) chlorophyll-based photosynthesis is carried out using the Qx and Qy (600-1000 nm) first excited singlet state of a small fraction of the total (bacterio-) chlorophyll in a cell. The Bx and By (420$470 \mathrm{~nm}$ ) second excited singlet states of (bacterio-) chlorophyll do not themselves perform photochemistry but energize photochemistry by internal conversion to the Qx and Qy excited states. The bulk of the (bacterio-) chlorophylls in a cell, as well as the carotenoids and phycobilins, are involved in photosynthesis by absorbing radiation and handing on the excitation energy to the (bacterio-) chlorophylls which do perform photochemistry. An example is shown by Herek et al. (2002) (their Fig. 1) of how this ultra-fast energy transfer takes place 
between carotenoid and bacteriochlorophyll molecules in the purple bacterium R. acidophilia.

\section{Spectral Mismatch Between Solar Radiation and the Absorption Spectra of Photosynthetic Pigments}

More than $99 \%$ of photosynthesis on Earth today involves chlorophyll based photochemistry, with absorption maxima for the photochemically active pigments at about $440 \mathrm{~nm}$ and $680 \mathrm{~nm}$ (see Falkowski \& Raven 1997). Just over half of this photosynthesis occurs on land in organisms with a trough in their absorption spectrum in the $500-600 \mathrm{~nm}$ range. This trough is not as evident in the intact cell as in extracted chlorophyll solutions due to the large amount of chlorophyll per unit area exposed to solar radiation. Nevertheless, the solar radiation reaching the Earth's surface peaks at about $550 \mathrm{~nm}$, so there is a major spectral mismatch. This conundrum has been discussed by many, notably Wald (1974). How has this mismatch happened?

The evolution of photosynthesis began in the oceans, and almost half of the photosynthesis on Earth today occurs in the sea. Marine photosynthetic organisms as a whole have a wider range of photosynthetic pigments than do those on land, and the maximum wavelength of radiation incident on organisms in open ocean water is at a shorter wavelength than on land since the spectral absorption of water (see e.g., Hale \& Querry 1973) reaches a minimum in the blue $(\sim 420 \mathrm{~nm})$. Further, water preferentially absorbs UV-B radiation relative to the shorter wavelengths of visible radiation and it has been suggested that photosynthesis evolved deep in water since it acted as a UV-B screen. The need for such a screen comes from the higher ratio of UV-B to longer wavelengths of radiation earlier in the sun's existence, and the absence of an ozone screen before the net generation of $\mathrm{O}_{2}$ in marine photosynthesis had established an oxygenated atmosphere.

According to this scenario the main non-damaging wavelengths of solar radiation which would have been available for photosynthesis for the earliest photosynthetic organisms would have been in the 400-500 nm range, corresponding to the $\mathrm{Bx}$ and $\mathrm{By}$ absorption bands of (bacterio-) chlorophyll. The rapid conversion ( p picosecs) of the second excited singlet state to the longer-lived first excited singlet state, corresponding to the Qx and Qy absorption bands, would favour the use of the first excited state in photochemistry. By the time that the UV-B flux incident at the land or water surface had decreased to levels which permitted photosynthetic (and other) organisms to occur at the sea surface or on land, the role of (bacterio-) chlorophylls as the photochemical catalysts in photosynthesis were evolutionarily well entrenched. From this point of view the spectral mismatch is a result of the spectral properties of cyclic tetrapyrroles which were and are chemically stable, had blue radiation-absorbing properties appropriate for photosynthesis deep in the ocean, and performed photochemistry using an excited state which was not significantly generated by the direct absorption of long wavelength radiation deep in the ocean. Accordingly, the spectral mismatch of photosynthesis at the sea or land surface can be regarded as an evolutionary accident of the habitat in which photosynthesis evolved. Note that Wald's (1974) comment that the ocean environment on the early Earth (i.e. 
its chemistry) may also have played a role, namely in the choice of $\mathrm{Mg}$, rather than say $\mathrm{Ca}$ or $\mathrm{Zn}$, as the central metal ion of the chlorophyll pigments on Earth, and that a similar choice may have been likely on other planets. Wald (1974) argues that the extreme stability of the porphyrin structure itself makes the chlorophyll pigment a "natural" choice for pigments beyond the Earth.

\section{Earth-Like Planets (ELPs)}

The (as yet hypothetical) ELPs could be orbiting "hot" (A, F) type or "cool" $(\mathrm{K}, \mathrm{M})$ type main sequence stars, as well as $\mathrm{G}$ type stars similar to the Sun. The spectral distribution of energy reaching the ELP varies greatly among these different stellar spectral types.

For "hot" stars there is no great impediment for photosynthesis to operate using pigments spectrally similar to those found on Earth. The high flux of damaging UV-B radiation from the "hot" stars can be handled, provided screening by ocean waters is an option at the earliest epochs; and later, assuming that atmospheric oxygenation by marine photosynthesis, as well as the burial of reduced carbon has taken place, an atmospheric ozone layer would form and allow photosynthesis to occur on land also. The spectral distribution of photosynthetically usable radiation from such stars matches well with the absorbance minimum of pure water and the absorbance maxima of chlorophyll ( $\mathrm{Bx}$ and $\mathrm{By}$ bands) and the carotenoids. Photosynthesis associated with "hot" stars would of course have had to evolve rapidly because of the limited time that such stars spend on the main sequence before the conditions turned harsh.

The evolution of photosynthesis on ELPs orbiting "cool" stars may be more problematic, at least when the UV and blue radiation fluxes are considered This is because the variable flux in the UV-B, associated with chromospheric activity and heating of magnetic origin, makes a much greater proportional contribution to the total flux in cool as opposed to hot stars. While the steady (photospheric) UV flux radiated outside the active phase is relatively small, so allowing photosynthesis to occur in the surface ocean or on land without the need for an atmospheric UV screen, the predominance of cool star radiation at longer wavelengths (ie near the $670 \mathrm{~nm}$ peak of Chla) means that the strong red absorption by water is a major obstacle to photosynthesis deep in the ocean. Although accurate statistics on the chromospheric/flare activity in the UV-B of cool and relatively young stars are hard to come by (see e.g., Ayres et al. 1996) it appears that this activity is primarily at wavelengths below $200 \mathrm{~nm}$ in solar type stars in young galactic clusters such as the Pleiades (70 Myr) and the Hyades (600 Myr old). Such short wavelength UV is unlikely to have reached the surface given the strong absorption by carbon dioxide below $220 \mathrm{~nm}$ which would probably have been present in the ELP atmosphere. Photosynthetic machinery which involves photochemically active pigments using excited states corresponding to near-infrared wavelengths $(\sim 700-1000 \mathrm{~nm})$ would require a three photon mechanism to move an electron from water to carbon dioxide rather than the two photon mechanism used on Earth (Wolstencroft \& Raven 2002). However for stars in the youngest active $\mathrm{T}$ Tauri phase (at $<10 \mathrm{Myr} \mathrm{BP}$ ), large temporal (months/years) variations in UV-B flux flux are likely which would involve periods during which photosynthesis would be restricted to considerable depths in the ocean at which UV-B is largely screened out, using UV-A and blue photons 
for profitable photochemistry. During flares photosynthetic activity could involve UV absorption by proteins with excitation energy transfer to chlorophylls which perform the photochemistry using the UV-derived energy plus blue photons which the chlorophyll has absorbed. This has already been suggested for the evolution of photosynthesis on Earth during the steady high surface UV flux with a young Sun and no atmospheric ozone as a UV screen. Such a scheme would require a 'feast and famine' existence of photosynthetic organisms deep in the ocean during the flare epoch, but there are precedents for this on an annual basis for high-latitude populations on Earth today. Evolution of photosynthesis during the flare epoch could, by the early involvement of photochemically active pigments with spectral properties similar to those used in oxygen-evolving photosynthesis on Earth, give rise to a two photon mechanism of photosynthesis during the post-flare phase which, for historical evolutionary reasons, also showed a spectral mismatch of the pigments and the incident radiation at the sea surface and on land.

\section{Summary}

The apparent spectral mismatch between solar radiation at the Earth's surface (peaking at $550 \mathrm{~nm}$ ) and the two absorption maxima (440 nm and $680 \mathrm{~nm}$ ) of chlorophyll pigments can be explained as an evolutionary accident of the marine habitat in which photosynthesis evolved on Earth .A similar scenario is likely for some ELPs due to certain universals such as the spectral absorption properties of water; and the geometric rigidity and great stability of chlorophyll, which makes it a likely "universal pigment"; provided the ELP ocean is of sufficient volume and depth to permit (a) protection from damaging UV-B radiation at the earliest epochs; and (b) the later generation of an oxygenated atmosphere together with an ozone screen.

The effects of variable UV emission, particularly in the UV-B, would cause relatively few problems in the evolution of photosynthesis for young $A, F$ and $\mathrm{G}$ stars, but could be a major problem for cool $\mathrm{K}$ and $\mathrm{M}$ stars for which UV variability causes major changes of the total UV flux and prevents efficient use of most of its photon flux because of the high absorption of red light by water. A 'feast and famine' existence for photosynthetic organisms in the deep ocean may work but would not be very productive. When UV variability has eventually died out on cool stars photosynthetic machinery involving photochemically active pigments at near-IR wavelengths $(\sim 700-1000 \mathrm{~nm})$ using a three photon mechanism would be needed to fully exploit the photons falling beyond the $700 \mathrm{~nm}$ limit of the two-photon terrestrial mechanism.

\section{References}

Ayres, T. R., Simon, T., Stauffer, J. R., Stern, R. A., Pye, J. P., \& Brown, A. 1996, ApJ, 473, 279

Falkowski, P. G., \& Raven, J. A. 1997, in Aquatic Photosynthesis (Massachusetts: Blackwell Science), 54

Hale, G. M., \& Querry, M. R. 1973, Appl.Optics, 12, 555

Herek, J. L. et al. 2002, Nature, 417, 533

Wald, G. 1974, Origins of Life, 5, 7

Wolstencroft, R. D., \& Raven, J. A. 2002, Icarus, 157, 535 